\title{
Probe into the Problem of Airport Taxi Queuing
}

\author{
Ouyang Liang ${ }^{1}$, Pan Huilin², Wang Erkang ${ }^{3}$ \\ ${ }^{1}$ School of Communication and Engineering, Hubei University of Economics, Wuhan 430205, China \\ ${ }^{2}$ Department of Accounting, School of Law and Business, Hubei University of Economics, Wuhan 430205, \\ China \\ ${ }^{3}$ School of Information and Communication Engineering, Hubei University of Economics, Wuhan 430205, \\ China
}

Keywords: difference equation factor analysis genetic algorithm

\begin{abstract}
Taxi is one of the main means of transportation for airport passengers. Taxi drivers are faced with two choices at the airport: "waiting in line to pick up passengers" and "directly emptying". This article studies the factors that influence the decision-making of taxi drivers, considering the profit of the driver and the number of passengers in other circumstances, provide suggestions for the taxi driver's decision-making, consider arranging a reasonable "boarding point" for passengers, and give short-distance drivers their corresponding "priority" to ensure a balanced income. We accurately calculate the costs and benefits based on the assumptions of rational people, and use the sum of the queue time and the return time in the queue waiting mode as the time standard, and compare the two modes of waiting in line and returning empty cars during this period of time. Profit, and thus choose the most profitable model. We collected relevant data on Wuhan Tianhe Airport and taxis in Wuhan by consulting relevant information. After the solution is solved, the rationality of the model is analyzed from the perspectives of hypothesis, logic and reality, and the factor analysis of the nine factors mentioned in question 1 is carried out using spss software. We considered two different modes of getting on the car. We take the time it takes for all taxis to carry passengers in the first mode as the standard, give priority to all passengers in the time period, and use the standard time that can just be met in both modes as the critical point. Finally, we adopt the "time compensation" method, we introduce the maximum journey as a reference, and give different time compensation to taxis of different distances.
\end{abstract}

\section{Problem restatement}

Most passengers go to the city (or surrounding) destinations after getting off the plane, and taxis are one of the main means of transportation. Most domestic airports separate the drop-off (departure) and pick-up (arrival) channels. Taxi drivers who drop off passengers to the airport will face two choices:

(1) Go to the arrival area and wait in line to take passengers back to the city. Taxis must wait in line at the designated "car storage pool" and enter the venue in a "first come, first come" queue to pick up passengers. The waiting time depends on the number of taxis and passengers in the queue, and requires a certain time cost.

(2) Directly vacate and return to the city to solicit customers. Taxi drivers will pay no-load charges and may lose potential passenger revenue.

The number of flights arriving in a certain period of time and the number of vehicles already in the "car storage pool" are certain information that the driver can observe. Usually the driver's decision is related to his personal experience judgment, such as the number of flights arriving in a certain season and time period and the number of possible passengers. If passengers want to "take a taxi" after disembarking the plane, they must queue up at the designated "ride area" and board in order. Airport taxi management personnel are responsible for letting taxis into the "riding area" in "quantitative batches" and arranging a certain number of passengers to board the bus. In practice, there are still many deterministic and uncertain factors that affect taxi drivers' decision-making, and their correlations are different, and their effects are also different. 


\section{Problem analysis}

First of all, we must analyze and study the factors related to the decision-making of the taxi driver and the influence of these factors on the driver's decision-making. For the decision-making model of the taxi driver, it is necessary to compare the expected revenue and the driver during the period of time when the driver chooses to queue up and return to the city Choose the empty car to return to the expected return in the market, that is, make a decision by comparing the return of the two choices in the same time

It is required to collect relevant data about taxis in a certain domestic airport and the city where it is located. Relevant data can be inquired through the Internet, or data mining can be carried out through computer software, and the rationality analysis of the airport taxi driver's selection plan and model for the data checked In fact, it is the visualization of the problem one model and solution, and the dependence on related factors can be analyzed and integrated with the related factors mentioned in the problem one through factor analysis.

The third problem is to design a "boarding point" to make the ride efficiency the most. For different "boarding points", it means different boarding modes, and two parallel lanes are given in this question, so two different boarding modes can be obtained, one is parking in two parallel lanes. Taxis are full, but in this case, because there is no lane for taxis to leave alone, the vehicles cannot leave alone with passengers, and only leave together when all the parked taxis are carrying passengers. The other is the parking space of one lane for taxis waiting in line for passengers, and the other lane is free for taxis carrying passengers to leave at any time. For the most efficient ride, since the number of passengers arriving in a unit time is the same, it is necessary to compare whether the two cases can pull all the passengers who come to ride the car within a unit time. If so, the second is Consider keeping each customer's waiting time as short as possible.

Since short-distance passengers can no longer carry passengers when they return to the airport, the problem requires that taxis returning short-distance passengers be given a certain "priority" so that the revenue of these taxis is as balanced as possible, and because of the randomness of passengers and the inability of drivers Therefore, the long-distance passenger-carrying driver cannot be punished, so you can consider the corresponding "compensation" to the driver according to the different journeys of each taxi carrying passengers: according to the requirement of income balance, it is derived that taxis carrying passengers of different distances The time that should be reduced and exempted separately, the "priority" of short-distance taxi drivers' time can be realized through the management of express channels.

\section{Model establishment and solution}

\subsection{Driver's decision model solution}

According to the requirements of the subject, we collected data on the status of taxis in Tianhe Airport and Wuhan on March 23, 2019, including: the number of vehicles waiting to be transported in the venue per minute, the number of vehicles entering the venue in the first half hour, the number of leaving vehicles in the first half hour, The number of new passengers, the number of new passenger groups, the number of people on the bus, and the number of people who did not get on the bus; the number of vehicles waiting to be transported in the city and the number of passenger vehicles in the city. (See supporting materials for detailed data) 


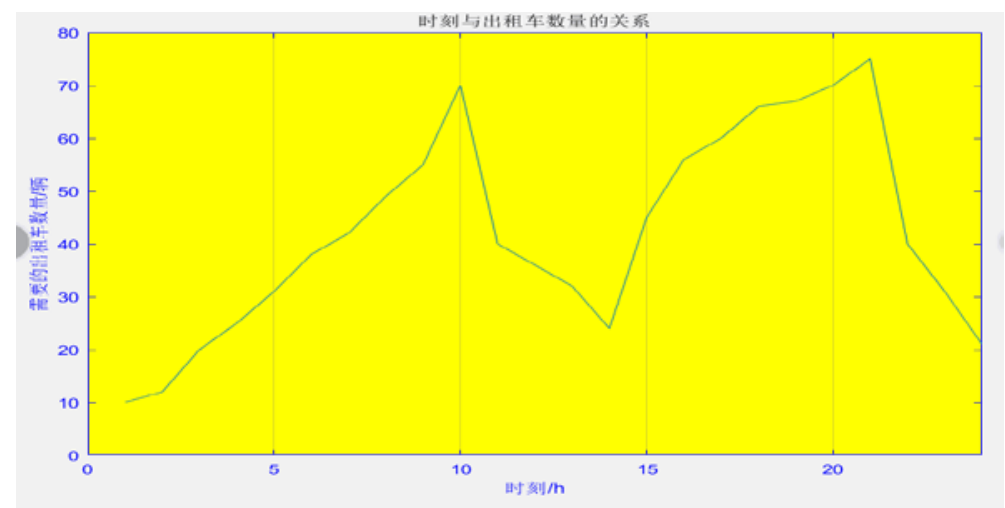

Figure 1 The relationship between time and the number of taxis

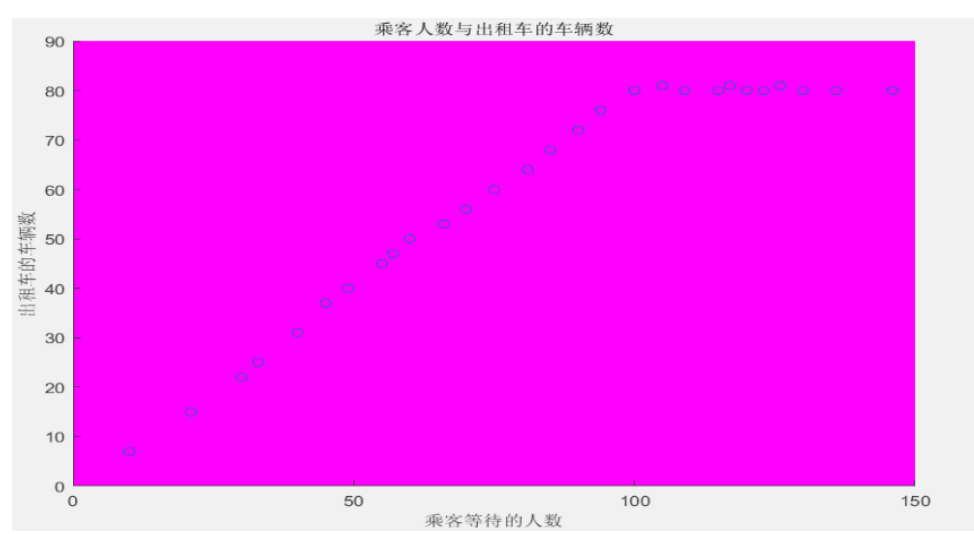

Figure 2 Number of passengers and number of taxi vehicles

Check the data and the fuel consumption per minute is 0.093 liters $/ \mathrm{km}$ 8 yuan per liter of oil

Suppose the expected return of mode $1 \mathrm{Y} 1=50$

Return time

$$
\mathrm{h}(\mathrm{t})=50
$$

According to the data calculated by Matlab:

Mode 1 queue time $=14$

Its cost

$$
\mathrm{C} 1=* 0.093 * 8=50 * 0.093 * 8=37.2
$$

Mode 2 return time

$$
g(t)=30
$$

Therefore, the city passenger time in Mode 2

$$
\mathrm{t}^{\prime \prime}=\mathrm{t}^{\prime}+\mathrm{h}(\mathrm{t})-\mathrm{g}(\mathrm{t})=14+50-30=34
$$

Its cost

$$
\mathrm{C} 2=(\mathrm{g}(\mathrm{t})+) * 0.093 * 8=47.616
$$

Its expected return

$$
\mathrm{Y} 2=\mathrm{k} \text {, }
$$

then

$$
\begin{array}{r}
\mathrm{Y} 2=1.5 * 34=51 \\
\mathrm{Y} 1-\mathrm{C} 1=50-37.2=12.8
\end{array}
$$




$$
\mathrm{Y} 2-\mathrm{C} 2=51-47.616=3.384
$$

Therefore, Y1- C1> Y2- C2, the driver should choose to wait in line.

First of all, the assumption of the model is reasonable, because for a taxi driver, maximizing the benefit is its biggest goal, so the assumption of a rational person is reasonable. Second, for a taxi, no matter how many trips there are in the car. Charges per person are the same, so the assumption that the number of passengers does not affect revenue is reasonable.

The operating process of this model is in line with reality, because when taxis are waiting in line for passengers, passengers need to have time to board the bus and there is a situation where multiple people in the queue go to the same destination together, so the passengers are divided into different groups according to batch Getting in the car is reasonable. Secondly, because the traffic conditions are different in each time period, it is in line with reality to make different assumptions about the time required for drivers to return to the city at different times.

The decision of this model is logical, because in order to find out which situation has the highest benefit, it can only be compared in the same time period, and it is reasonable to use the queue time and the return time as the time standard. Because this happens to be a complete cycle of queuing for passengers, the benefit gained during this time period is the opportunity cost of returning empty cars.

In response to the nine influencing factors mentioned in question 1, we randomly surveyed 20 taxi drivers, and each driver scored the nine factors. The higher the score, the more they will consider this factor when making decisions.

Since the nine factors are many and some overlap, we use spss software to perform factor analysis on the table.

\subsection{Taxi driver queuing model}

Assuming that every vehicle or group of vehicles in the storage tank leaves, the next vehicle or group of vehicles will automatically fill the seats, and the supply of taxis is large enough.

Assuming that each lane has $a$ parking spaces, the two-lane lane can accommodate up to $2 a$ taxis waiting in line to carry passengers.

Suppose the passenger flow to the queue at time $t$ is $m(t)$

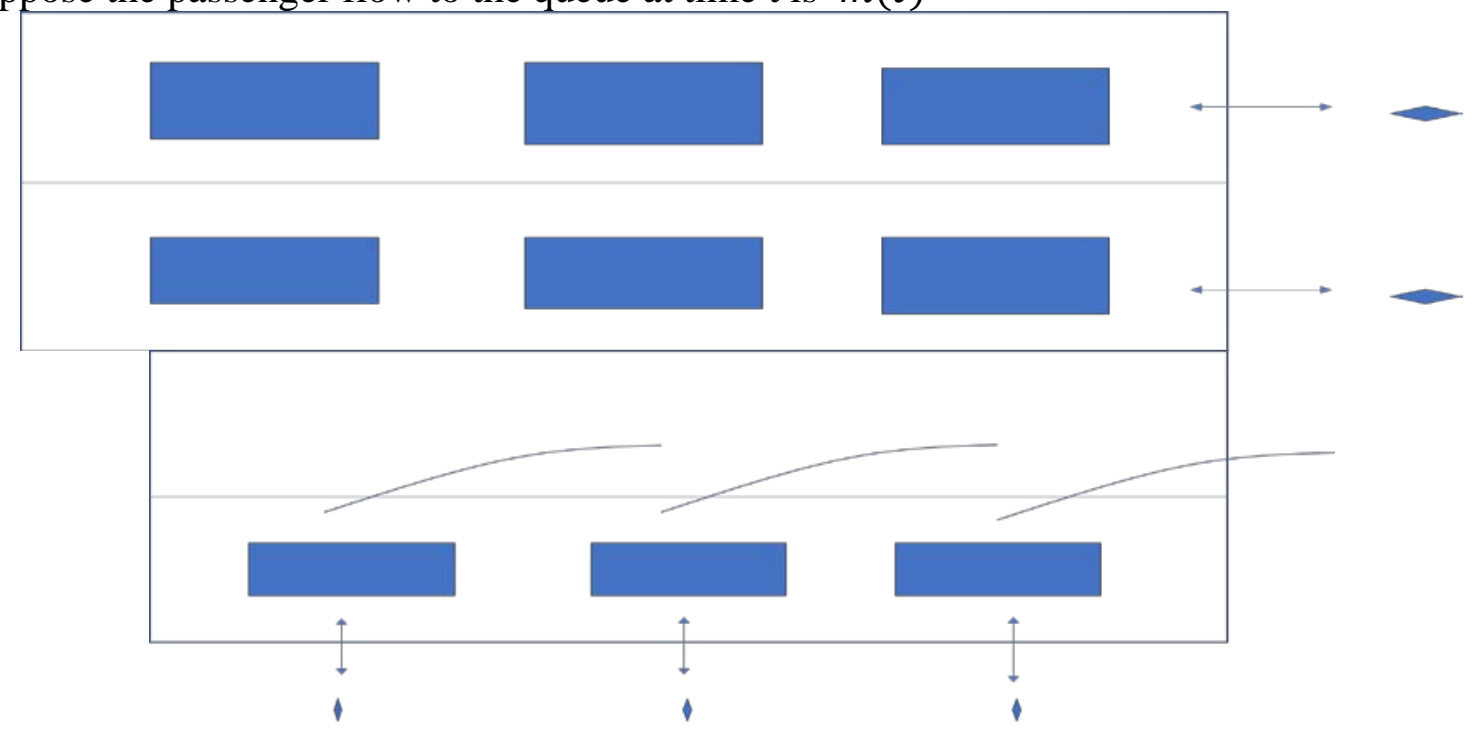

Figure 3 Multi-point parallel taxi queuing service system 1

\subsubsection{The first boarding mode:}

Suppose that in this mode, the time spent waiting for passengers in a queue to carry passengers is from $2 \mathrm{a}=\sum_{t=t_{i}}^{t_{i}+j} m(t)$ to $2 \mathrm{a}=\sum_{t=t_{i}}^{t_{i}+j} m(t)$

then 


$$
2 \mathrm{a}=\sum_{t=t_{i}}^{t_{i}+j} m(t)
$$

\subsubsection{The second boarding mode:}

In this mode, the taxi driver can pick up passengers and leave at any time, so it is necessary to assume that the time it takes for each passenger to board the bus is. In order to optimize the time to board the bus, we use genetic algorithm to get the optimal boarding time-min \{\} (See the MATLAB code in the appendix), and each lane has a pick-up point, so people can board at the same time within a certain period of time, and for each person in the vehicle, taking into account safety factors, after the previous vehicle has left There is a time to leave the safe distance and the time to make up for the car, which is assumed to be, therefore, the time used for the first mode to download passenger 2 in an ideal state during this time period (regardless of the number of people queuing in this time period), The number of passengers that can be carried is

$$
\frac{\left(t_{i+j}-t_{i}\right)}{\frac{s}{a}+s^{\prime}}
$$

In summary:

$$
\begin{aligned}
& \text { When } 2 a>\frac{\left(t_{i+j}-t_{i}\right)}{\frac{s}{a}+s^{\prime}}, \text {, select mode one. } \\
& \qquad 2 a \leq \frac{\left(t_{i+j}-t_{i}\right)}{\frac{s}{a}+s^{\prime}} \text {, select mode two. }
\end{aligned}
$$

\subsubsection{Assignment calculation}

Suppose the parking space in each lane $\mathrm{a}=10$

Assuming that in the second mode, the time for each passenger to board the vehicle $s=1 / 12$, and the time to leave the safe distance and the time to make up the car $s^{\prime}=1 / 6$, the substitution value is worth:

$$
2 a *\left(\frac{a}{s}+s^{\prime}\right)=2 * 10 *\left(\frac{\frac{1}{12}}{10}+\frac{1}{6}\right)
$$

When, $t_{i+j}-t_{i} \leq \frac{21}{6}$ select mode one.

When $t_{i+j}-t_{i}>\frac{21}{6}$, select mode two.

\subsection{Taxi compensation plan}

Taxi fares currently generally use a step function method, that is, within a kilometer, the charge is b yuan, and if the distance exceeds a kilometers, an additional c yuan will be charged for each kilometer. The specific function is as follows:

$$
y=\left\{\begin{array}{l}
b, s_{i} \leq a \\
b+\left(s_{i}-a\right) c, s_{i}>a
\end{array}\right.
$$

There are many taxi costs, and only the most important fuel costs are considered here: assuming that the fuel consumption per kilometer is $d$ yuan.

According to profit = income-cost, taxi profit can be expressed as: 


$$
Z_{i}=\left\{\begin{array}{l}
b-d^{*} s_{i}, s_{i} \leq a \\
b-d^{*} s_{i}+\left(s_{i}-a\right) * c, s_{i}>a
\end{array}\right.
$$

Since the amount of "compensation" for the taxi driver needs a reference object, the maximum mileage of the taxi is used as the reference object, so there is any mileage

$$
S_{i} \leq S_{m}
$$

According to time=distance/speed (assuming that the taxi is traveling at a constant speed):

$$
t_{m}=\frac{s_{m}}{v} t_{i}=\frac{s_{i}}{v}
$$

Let $\Delta$ tbe the difference between the maximum mileage time and any mileage time, then:

$$
\Delta t=t_{m}-t_{i}
$$

Due to the need to consider the balance of taxi revenue, considering that the probability of the driver picking up and returning is extremely low and does not need to be subsidized, we assume that all the returned taxis are not picked up, and we will make any taxi profit Evenly apportion the arrival and return mileage, passing the profit per unit of mileage $*$ the mileage of the taxi $=$ the difference between the maximum mileage profit and the profit of the mileage, which is the time that the taxi waits for passengers in the storage pool after being "compensated". Therefore, the following equation can be obtained:

$$
\frac{z_{i}}{2 s_{i}} *(\Delta t-t) * v=\mathrm{z}_{m}-z_{i}
$$

Solved:

$$
t=\left(1-\frac{2 s_{i}}{z_{i}}\right) *\left(t_{m}-t_{i}\right)
$$

Time reduction and exemption can be superimposed, and a recording system can be introduced to record the time reduction and exemption of taxis. Taxi drivers can choose whether to use the time reduction or exemption according to their own needs. Regarding the control of time reduction and exemption, you can manage the fast lane to make the choice of time reduction and exemption. The driver can wait for the time obtained by subtracting the time reduction from the original waiting time before being released.

\section{Analysis of advantages and disadvantages}

The advantage of solving the problem is that it accurately calculates the time the driver spends waiting in line, and accurately calculates the income and cost of the driver at the same time in the two cases, which maximizes the interests of the driver.

1) The advantage of solving problem two is

The advantage of solving problem three is that it not only considers the maximization of passengers per unit time, but also considers the minimization of the waiting time of each individual, which is convenient for different time boarding modes according to the changes of passenger flow over time. Switch.

The advantage of solving problem 4 is that it takes the equilibrium income as the core to model, the derivation process is relatively simple, and it ensures that the priority of taxis for different distances is also different.

The shortcoming of the first solution is that in the two cases, the time it takes for the driver to return to the city does not find a specific function of time, and it is a bit vague to rely solely on the 
driver's subjective judgment of the traffic situation. No specific functional relationship is given for the change of passenger flow over time, the time when passengers board the bus, and the relationship between return and time after returning to the market.

2) The disadvantage of solving problem two is

The shortcoming of problem three is that the safety factors in case two are not fully considered, which easily leads to traffic jams caused by vehicles leaving at the same time. Secondly, it is assumed that the supply of taxis is large enough, which is not very consistent with the actual situation. Furthermore, there is no specific functional relationship for the change of passenger flow over time and the time when passengers attend classes.

The shortcoming of the fourth problem is that there is a certain amount of compensation for long distances, so there is no priority for short distances, and the complicated traffic conditions are not considered, and the vehicle speed is directly assumed to be uniform.

\section{References}

[1] Jiang Qiyuan, Xie Jinxing, Ye Jun, Mathematical Model [M]. Beijing: Higher Education Press, 2004.

[2] Xue Dingyu, Chen Yangquan, MATLAB Solving of Advanced Applied Mathematics [M]. Beijing: Tsinghua University Press, 2004.

[3] Wei Zhonghua, Wang Lin, Qiu Shi. Optimization of the service desk in the taxi pick-up area in the hub based on queuing theory [J]. Highway Transportation Science and Technology (Application Technology Edition), 2017, 13(10): 298-300.

[4] $\mathrm{Hu}$ Cheng. Research on the parallel three-dimensional taxi queuing system. Urban roads, bridges and flood control, 2014(7): 19-21.

[5] Sun Rongheng, Li Jianping. The basis of queuing theory. Science Press, 2002.

[6] Hu Yunquan. Fundamentals and Application of Operations Research. Wuhan University Press, 2014.

[7] Li Dongping, Yan Kefei, Cheng Linjie, etc. Service level model of airport taxi pick-up area type. Journal of Harbin Institute of Technology, 2011, 43(4): 126-130.

[8] DA COSTA DCT, DE NEUFVILLE R. Designing Efficient Taxi Pick up Operations at Airports. Transportation Research Record, 2012, 2300(1): 91-99. 